\title{
The interplay among compositional heterogeneity, lattice defects, micromorphology, and redox stratification in lithium-ion batteries
}

Yijin Liu

SLAC National Accelerator Laboratory, Menlo Park, California, United States

Lithium-ion batteries (LIBs) are hierarchically structured, featuring multi-scale heterogeneities in micromorphology, composition, structure deformation, mechanical strain, and redox chemistry, all of which are closely associated with the ultimate battery functionality. It is of fundamental interest and practical importance to probe the battery material with high spatial resolution, sufficient chemical sensitivity, and covering a statistically representative volume. State-of-the-art synchrotron-based x-ray microscopy techniques are being broadly applied to battery research. Depending on the experimental configuration and on the types of signals that are harvested, the synchrotron techniques can probe many different aspects of the material property, e.g. lattice arrangement, micromorphology, and oxidation states. The novel developments in the synchrotron experimental techniques, however, bring in challenges associated with the unprecedented high data rate and complexity. With the aid of advanced computational developments, e.g. machine learning methods, it is now feasible to efficiently and effectively extract scientifically relevant information from large-scale synchrotron data. In synchrotron-based battery research, rapid extraction of useful information from the large data is key to revealing the electro-chemomechanical interplay in functioning lithium-ion batteries.

In this presentation, I will review my group's recent research activities in this field. We combined synchrotron-based multi-modal experimental tools with machine learning models that are tailored for our applications. At the mesoscale, we observe the strain-redox coupling and decoupling effects within single NMC cathode particles, which are attributed to different reaction mechanisms. At the electrode scale, we conduct statistical analysis on thousands of particles in a high-throughput fashion and formulate an understanding of the particle's chemomechanical behavior with statistical significance. Our results not only reveal important degradation mechanisms in LIBs but also can critically inform the design of nextgeneration LIBs with improved robustness.

\section{References}

[1] Guannan Qian, Jin Zhang, Sheng-Qi Chu, Jizhou Li, Kai Zhang, Qingxi Yuan, Zi-Feng Ma, Piero Pianetta, Linsen Li, Kee-young Jung, Yijin Liu, "Understanding the mesoscale degradation in nickel-rich cathode materials through machine-learning-revealed strain-redox decoupling," ACS Energy Lett. doi:10.1021/acsenergylett.0c02699 (2021).

[2] Zhisen Jiang, Jizhou Li, Yang Yang, Linqin Mu, Chenxi Wei, Xiqian Yu, Piero Pianetta, Kejie Zhao, Peter Cloetens, Feng Lin, Yijin Liu, "Machine-learning-revealed statistics of the particle-carbon/binder detachment in lithium-ion battery cathodes", Nature Communications 11, 2310 (2020).

[3] Jin Zhang, Qinchao Wang, Shaofeng Li, Zhisen Jiang, Sha Tan, Xuelong Wang, Kai Zhang, Qingxi Yuan, Sang-Jun Lee, Charles J. Titus, Kent D. Irwin, Dennis Nordlund, Jun-Sik Lee, Piero Pianetta, Xiqian Yu, Xianghui Xiao, Xiao-Qing Yang, Enyuan Hu, Yijin Liu, "Depth-dependent valence stratification driven by oxygen redox in lithium-rich layered oxide," Nature Communications 11, 6342 (2020).

[4] Yang Yang, Rong Xu, Kai Zhang, Sang-Jun Lee, Linqin Mu, Pengfei Liu, Cyrstal K. Waters, Stephanie Spence, Zhengrui Xu, Chenxi Wei, David J. Kautz, Qingxi Yuan, Yuhui Dong, Young-Sang Yu, Xianghui 
Xiao, Han-Koo Lee, Piero Pianetta, Peter Cloetens, Jun-Sik Lee, Kejie Zhao, Feng Lin, Yijin Liu, "Quantification of Heterogeneous Degradation in Li-ion Batteries," Adv. Energy Mater. 1900674, (2019). 